\title{
A SOR Acceleration of Self-Adjoint and m-Accretive Splitting Iterative Solver for 2-D Neutron Transport Equation
}

\author{
O. Awono ${ }^{1}$, J. Tagoudjeu ${ }^{1,2} *$ \\ ${ }^{1}$ ENSP, University of Yaoundé I, P.O. Box 8390 Yaoundé, Cameroon \\ ${ }^{2}$ Faculty of Science, University of Yaoundé I, P.O. Box 812 Yaoundé, Cameroon
}

\begin{abstract}
We present an iterative method based on an infinite dimensional adaptation of the successive overrelaxation (SOR) algorithm for solving the 2-D neutron transport equation. In a wide range of application, the neutron transport operator admits a Self-Adjoint and m-Accretive Splitting (SAS). This splitting leads to an ADI-like iterative method which converges unconditionally and is equivalent to a fixed point problem where the operator is a 2 by 2 matrix of operators. An infinite dimensional adaptation of a SOR algorithm is then applied to solve the matrix operator equation. Theoretical and numerical results of convergence are given.
\end{abstract}

Key words: neutron transport, operator splitting, self-adjoint, m-accretive, iterative methods, SOR acceleration

AMS subject classification: 82D77, 82C70, 47J25, 47B44, 65B99

\section{Introduction and mathematical setting}

This paper focus on developing iterative methods for the numerical treatment of the single group steady state first order neutron transport equation in 2-D cartesian geometry verified by the neutron flux $u(r, \omega): Q:=R \times B \rightarrow \mathbb{R}^{+}$, solution of:

$$
\left\{\begin{array}{l}
T u(r, \omega):=A u(r, \omega)+\Sigma u(r, \omega)-K u(r, \omega)=q(r, \omega), \quad(r, \omega) \in Q \\
u(r, \omega) \in D(T)
\end{array}\right.
$$

${ }^{*}$ Corresponding author. E-mail: jtagoudjeu@gmail.com 
with operators $A, \Sigma$ and $K$ defined by:

$$
A u=\omega \nabla_{r} u ; \quad \Sigma u=\sigma(r) u ; \quad K u=\int_{B} \kappa\left(r, \omega, \omega^{\prime}\right) u\left(r, \omega^{\prime}\right) d \omega^{\prime},
$$

and,

$$
D(T)=\left\{u \in L^{2}(Q): \omega \nabla_{r} u \in L^{2}(Q) \text { and } u_{\mid \Gamma_{-}}=0\right\},
$$

where $\Gamma_{-}=\left\{r \in \partial R \times B: \mu n_{x}+\eta n_{y}<0\right\}\left(n=\left(n_{x}, n_{y}\right)\right.$ being the outer normal to $\left.\partial R\right)$, $r=(x, y), \omega=(\mu, \eta), R=] 0,1[\times] 0,1\left[\right.$ and $B=\left\{\omega \in \mathbb{R}^{2}:|\omega|<1\right\}$. The function $\sigma(r)$ represents the total cross section and $\kappa\left(r, \omega, \omega^{\prime}\right)$ is a non negative kernel describing the scattering of particles. The function $q$ is the non negative source term. We equip the space $D(T)$ with the norm

$$
\|u\|_{D(T)}^{2}=\|u\|^{2}+\left\|\omega \cdot \nabla_{r} u\right\|^{2}
$$

where $\|$.$\| is the standard L^{2}(Q)$ norm. Let $\alpha$ be a positive constant. The norm $\|\cdot\|_{\alpha}$ is defined in $D(T)$ by

$$
\|u\|_{\alpha}=\|(A+\alpha I) u\|
$$

where $I$ is the identity operator in $L^{2}(Q)$. This norm is equivalent to norm $\|\cdot\|_{D(T)}$, defined by (1.4) [1].

In the following, it is assumed that: (a) $\sigma \in L^{\infty}(Q)$ and $\exists \sigma_{0}>0$ such that $\sigma(r) \geq \sigma_{0}$ a.e. on $Q$; (b) $\kappa\left(r, \omega, \omega^{\prime}\right)=\kappa\left(x, \omega^{\prime}, \omega\right)$ and $\kappa$ is positive; (c) $\exists c \in[0,1), \int_{B} \kappa\left(r, \omega, \omega^{\prime}\right) d \omega^{\prime} \leq \sigma_{0} c$ a.e. on $Q$. The operator $A$ is m-accretive and following the above assumptions, the operator $S=\Sigma-K$ is self-adjoint and positive definite. Thus $T$ is positive definite and it follows the existence and uniqueness of the solution of problem (1.1). Moreover, $T$ admits a self-adjoint and $m$-accretive splitting (SAS) which yields the SAS iteration method.

For $\alpha>0$, the SAS iteration method for solving (1.1) is defined as follows: given $u^{0} \in D(T)$,

$$
\left\{\begin{array}{l}
(\alpha I+S) u^{\left(k+\frac{1}{2}\right)}=(\alpha I-A) u^{(k)}+q \\
(\alpha I+A) u^{(k+1)}=(\alpha I-S) u^{\left(k+\frac{1}{2}\right)}+q
\end{array} .\right.
$$

Let $u^{*}$ be the exact solution of (1.1). We have for any $k>0$,

$$
\left\|u^{*}-u^{(k+1)}\right\|_{\alpha} \leq\|M(\alpha)\|\left\|u^{*}-u^{(k)}\right\|_{\alpha}
$$

where $M(\alpha)=S_{1}(\alpha) A_{1}(\alpha)$, with $S_{1}(\alpha)=(\alpha I-S)(\alpha I+S)^{-1}$ and $A_{1}(\alpha)=(\alpha I-A)(\alpha I+A)^{-1}$. Let $\sigma(S)$ denotes the spectrum of $S$. It is proved [2,3] that $\left\|A_{1}(\alpha)\right\| \leq 1$ and $\left\|S_{1}(\alpha)\right\| \leq \beta(\alpha)<$ 1 , where $\beta(\alpha)=\sup _{\lambda \in \sigma(S)}\left|\frac{\alpha-\lambda}{\alpha+\lambda}\right|$ and, the theoretical optimal parameter $\alpha^{*}$ for the bound $\beta(\alpha)$ is $\alpha^{*}=\sqrt{\lambda_{\min } \lambda_{\max }}$ with $\lambda_{\min }$ and $\lambda_{\max }$ denoting respectively the lower and upper bound of $\sigma(S)$. It then follows that, $\|M(\alpha)\|<1$. For any $u^{(0)} \in D(T)$ and $k>0$,

$$
\left\|u^{*}-u^{(k)}\right\|_{\alpha}<[\beta(\alpha)]^{k}\left\|u^{*}-u^{(0)}\right\|_{\alpha} .
$$

Thus the SAS iteration (1.6) converges unconditionally to the solution of (1.1) with respect to norm $\|\cdot\|_{\alpha}$ and $\|\cdot\|_{D(T)}$. Since for $u \in D(T)$, we have $\|u\| \leq\|u\|_{D(T)}$, the convergence of the SAS 
iteration with respect of the standard $L^{2}(Q)$ follows. The convergence analysis of the incomplete version of SAS iteration where each subproblem of (1.6) is solved approximately, is given in [2]. The remainder of this paper is structured as follows: we give the description and the convergence properties of the SOR acceleration of the SAS iteration in Section 2. A bound for the spectral radius of the Jacobi iteration operator which depends solely on the spectrum of the self-adjoint part of the transport operator is derived. This bound allows the approximation of the optimal convergence parameter of the SOR method when the eigenvalues of the Jacobi iteration operator are real numbers. The numerical illustrations are presented in Section 3. Concluding remarks are given in Section 4.

\section{The SOR acceleration of the SAS iteration method.}

The following fixed point equation can be derived from the definition of the SAS iteration (1.6):

$$
\mathbf{T}(\alpha) \mathbf{u}=\mathbf{q}
$$

where $\mathbf{T}(\alpha)=\left(\begin{array}{cc}(\alpha I+S) & -(\alpha I-A) \\ -(\alpha I-S) & (\alpha I+A)\end{array}\right), \quad \mathbf{u}=\left(\begin{array}{c}u_{1} \\ u_{2}\end{array}\right)$ and $\mathbf{q}=\left(\begin{array}{c}q \\ q\end{array}\right)$.

For $\alpha>0$, the operators $(\alpha I+A)$ and $(\alpha I+S)$ are positive definite. It follows that $(\alpha I+A)^{-1}$ and $(\alpha I+S)^{-1}$ exist and it holds that

$$
\mathbf{T}(\alpha)=\left(\begin{array}{cc}
I & 0 \\
-(\alpha I-S)(\alpha I+S)^{-1} & I
\end{array}\right)\left(\begin{array}{cc}
(\alpha I+S) & -(\alpha I-A) \\
0 & A(\alpha)
\end{array}\right)
$$

with $A(\alpha)=(I-M(\alpha))(\alpha I+A)$. Since $\|M(\alpha)\|<1,(I-M(\alpha))^{-1}$ and $A(\alpha)^{-1}$ are bounded. It then follows the existence and the uniqueness of the solution of (2.1).

Let $\mathbf{u}=\left(u_{1}, u_{2}\right)^{t} \in H \times H$. We consider in $H \times H$ the norm $\|\mid \mathbf{u}\|\left\|^{2}=\right\| u_{1}\left\|^{2}+\right\| u_{2} \|^{2}$. Let $u^{*}$ be the solution of (1.1) and, let $\mathbf{u}^{*}=\left(u^{*}, u^{*}\right)^{t}$. For $\alpha>0$, we have $\mathbf{T}(\alpha) \mathbf{u}^{*}=\left((\alpha I+S) u^{*}-(\alpha I-A) u^{*},-(\alpha I-S) u^{*}+(\alpha I+A) u^{*}\right)^{t}=\left(T u^{*}, T u^{*}\right)^{t}=\mathbf{q}$.

Thus $\mathbf{u}^{*}$ is the solution of (2.1). Conversely, let $\mathbf{u}^{*}=\left(u^{*}, v^{*}\right)^{t}$ be the solution of (2.1). The functions $u^{*}$ and $v^{*}$ satisfy $(\alpha I+S) u^{*}-(\alpha I-A) v^{*}=q$ and $-(\alpha I-S) u^{*}+(\alpha I+A) v^{*}=q$. Through subtraction and substitution, we obtain $v^{*}=u^{*}$ and $T u^{*}=q$. It follows that problems (1.1) and (2.1) are equivalent.

Let consider in $\mathcal{D}(T) \times \mathcal{D}(T)$ the operator

$$
\mathbf{P}(\alpha)=\left(\begin{array}{cc}
(\alpha I+S) & 0 \\
0 & (\alpha I+A)
\end{array}\right) .
$$

The preconditioning of the system (2.1) from the right by $[\mathbf{P}(\alpha)]^{-1}$ leads to the following system

$$
\mathbf{T}_{1}(\alpha) \mathbf{u}=\mathbf{q},
$$

where $\mathbf{T}_{1}(\alpha)=\left(\begin{array}{cc}I & -A_{1}(\alpha) \\ -S_{1}(\alpha) & I\end{array}\right)$. The operator $\mathbf{T}_{1}(\alpha)$ is bounded and the solution $\mathbf{v}^{*}(2.1)$ reads $\mathbf{v}^{*}=[\mathbf{P}(\alpha)]^{-1} \mathbf{u}^{*}$, where $\mathbf{u}^{*}$ is solution of (2.3). 
The Jacobi method for the solution of (2.3) proceeds as follows: Let $\mathbf{u}^{(0)} \in H \times H$ be an initial guest for $k \geq 0$,

$$
\mathbf{u}^{(k+1)}=\mathbf{J}(\alpha) \mathbf{u}^{(k)}+\mathbf{q}
$$

where $\mathbf{J}(\alpha)=\left(\begin{array}{cc}0 & A_{1}(\alpha) \\ S_{1}(\alpha) & 0\end{array}\right)$. The corresponding SOR iteration with the relaxation parameter $\theta>0$, is

$$
\mathbf{u}^{(k+1)}=\mathbf{L}_{\theta}(\alpha) \mathbf{u}^{(k)}+\mathbf{q}_{\theta}(\alpha)
$$

where $\mathbf{L}_{\theta}(\alpha)=\left(\begin{array}{cc}(1-\theta) I & \theta A_{1}(\alpha) \\ \theta(1-\theta) S_{1}(\alpha) & (1-\theta) I+\theta^{2} M(\alpha)\end{array}\right)$. The choice of $\theta=1$ in the method (2.5) results in the Gauss-Seidel iteration for solving the system (2.3).

Proposition 1. Let $\alpha$ be a positive constant. It holds that:

$$
\begin{aligned}
\||J(\alpha)|\| & <1, \\
\left\|\left|L_{1}(\alpha)\right|\right\| & <\beta(\alpha) .
\end{aligned}
$$

It the follows that the Jacobi and Gauss-Seidel iteration methods for the solution of (2.3) converge. Moreover, the Gauss-Seidel and SAS iterations have the same bound for their contraction factors.

Proof. For $\mathbf{u}=\left(u_{1}, u_{2}\right)^{t} \in L^{2}(\Omega) \times L^{2}(\Omega)$,

$$
\||J(\alpha) \mathbf{u}|\|^{2}=\left\|A_{1}(\alpha) u_{2}\right\|^{2}+\left\|S_{1}(\alpha) u_{1}\right\|^{2} \leq\left\|u_{2}\right\|^{2}+\beta^{2}(\alpha)\left\|u_{1}\right\|^{2}<\||\mathbf{u}|\|^{2} .
$$

It follows that $\||J(\alpha)|\|<1$ and the Jacobi iteration method (2.4) converges.

Let $\mathbf{u}^{*}=\left(u_{1}^{*}, u_{2}^{*}\right)^{t}$ be the exact solution to $(2.3)$ and $\mathbf{u}^{(k)}=\left(u_{1}^{(k)}, u_{2}^{(k)}\right)^{t}$ be the $k^{t h}$ iterate obtained from the Gauss-Seidel iteration. We have

$$
u_{1}^{(k+1)}-u_{1}^{*}=A_{1}(\alpha) S_{1}(\alpha)\left[u_{1}^{(k)}-u_{1}^{*}\right] \text { and } u_{2}^{(k+1)}-u_{2}^{*}=S_{1}(\alpha) A_{1}(\alpha)\left[u_{2}^{(k)}-u_{2}^{*}\right] .
$$

Since $\left\|A_{1}(\alpha) S_{1}(\alpha)\right\| \leq \beta(\alpha)$ and $\left\|S_{1}(\alpha) A_{1}(\alpha)\right\| \leq \beta(\alpha)$,

$$
\left\|u_{1}^{(k+1)}-u_{1}^{*}\right\| \leq \beta(\alpha)\left\|u_{1}^{(k)}-u_{1}^{*}\right\| \text { and }\left\|u_{2}^{(k+1)}-u_{2}^{*}\right\| \leq \beta(\alpha)\left\|u_{2}^{(k)}-u_{2}^{*}\right\| .
$$

Therefore, the solution $\mathbf{u}^{*}$ verifies

$$
\left\|\left|\mathbf{u}^{(k+1)}-\mathbf{u}^{*}\right|\right\|=\left\|\left|L_{1}(\alpha)\left(\mathbf{u}^{(k)}-\mathbf{u}^{*}\right)\right|\right\| \leq \beta(\alpha)\left\|\left|\mathbf{u}^{(k)}-\mathbf{u}^{*}\right|\right\|<\left\|\left|\mathbf{u}^{(k)}-\mathbf{u}^{*}\right|\right\| .
$$

It follows that $\left\|\left|L_{1}(\alpha)\right|\right\| \leq \beta(\alpha)<1$ and the Gauss-Seidel iteration method converges.

Proposition 2. [1] Assume that $\theta \neq 0$. If $\lambda \in \sigma\left(\mathbf{L}_{\theta}(\alpha)\right) \backslash\{0\}$ and if $\tau$ satisfies

$$
(\lambda+\theta-1)^{2}=\lambda \theta^{2} \tau^{2}
$$

then $\tau \in \sigma(\mathbf{J}(\alpha))$. Conversely, if $\tau \in \sigma(\mathbf{J}(\alpha))$ and if $\lambda$ satisfies (2.8), then $\lambda \in \sigma\left(\mathbf{L}_{\theta}(\alpha)\right)$. 
The relation (2.8) between the spectrums of the operators of the Jacobi and SOR methods is the same as in the presence of finite dimensional linear systems with coefficient matrices possessing block Property A. Therefore, the results obtained in the last case may be generalized for the SOR acceleration of the SAS method. In particular, setting $\theta=1$ in (2.8), we have theoretically that Jacobi and Gauss-Seidel methods converge simultaneously and the Gauss-Seidel method is two times faster than Jacobi method. We also have the following convergence results of the SOR method $[4,5]$.

Proposition 3. 1. If $\sigma(\mathbf{J}(\alpha) \subset \mathbb{R}$, the SOR method converges for $0<\theta<2$ and the optimal convergence parameter is

$$
\theta_{\text {opt }}=\frac{2}{1+\sqrt{1-(r(\mathbf{J}(\alpha)))^{2}}},
$$

where $r(\mathbf{J}(\alpha))$ is the spectral radius of $\mathbf{J}(\alpha)$.

2. If $\sigma(\mathbf{J}(\alpha))$ contains complex numbers, the SOR method converges if for some positive number $\tau \in(0,1)$ and each $\lambda=\mu+i \beta \in \sigma(\mathbf{J}(\alpha))$, the point $(\mu, \beta)$ lies in the interior of the ellipse $\epsilon(1, \tau)=\left\{(\mu, \beta): \mu^{2}+\frac{\beta^{2}}{\tau^{2}}=1\right\}$ and $0<\theta<\frac{2}{1+\tau}$. Theoretical results for the determination of the SOR optimal parameter are given in [5].

Remark 4. From relations (2.7) and (2.8), we deduce that the spectral radius of $\mathbf{J}(\alpha)$ verifies

$$
r(\mathbf{J}(\alpha))<\sqrt{\beta(\alpha)}
$$

Therefore, if $\sigma(\mathbf{J}(\alpha)) \subset \mathbb{R}$, the optimal SOR parameter can be approximated by $\theta_{a}=\frac{2}{1+\sqrt{1-\beta(\alpha)}}$.

\section{Numerical results}

We took particular data for which an exact solution is known in each case. The iterations are stopped when the relative error $\left\|U-U_{\text {exact }}\right\|_{2} /\left\|U_{\text {exact }}\right\|_{2}<\epsilon(\epsilon>0)$.

$R=] 0,1[\times] 0,1\left[\right.$; for $x=\left(x_{1}, x_{2}\right) \in R$ and $\Omega=(\mu, \eta) \in B$, we set $\sigma(x)=\sigma, \kappa\left(x, \Omega, \Omega^{\prime}\right)=\frac{\sigma c}{\pi}$ and

$$
q(x, \mu)=\left\{\begin{array}{l}
\mu x_{2}+\eta x_{1}+\sigma x_{1} x_{2}-\frac{\sigma c}{4} ; \mu>0, \eta>0, \\
-\mu x_{2}+\eta\left(1-x_{1}\right)+\sigma\left(1-x_{1}\right) x_{2}-\frac{\sigma c}{4} ; \mu<0, \eta>0, \\
-\mu\left(1-x_{2}\right)-\eta\left(1-x_{1}\right)+\sigma\left(1-x_{1}\right)\left(1-x_{2}\right)-\frac{\sigma c}{4} ; \mu<0, \eta<0 \\
\mu\left(1-x_{2}\right)-\eta x_{1}+\sigma x_{1}\left(1-x_{2}\right)-\frac{\sigma c}{4} ; \mu>0, \eta<0 .
\end{array}\right.
$$

The exact solution of this test problem is given by:

$$
\psi(x, \mu)=\left\{\begin{array}{l}
x_{1} x_{2}, \quad \mu>0 \eta>0 ;\left(1-x_{1}\right) x_{2}, \quad \mu<0 \eta>0 \\
\left(1-x_{1}\right)\left(1-x_{2}\right), \quad \mu<0 \eta<0 ; x_{1}\left(1-x_{2}\right), \quad \mu>0 \eta<0 .
\end{array}\right.
$$

The spatial and angular discretization are carried out by a difference method based on volume control and a quadrature formula respectively. The spatial mesh size is $h_{x_{1}}=h_{x_{2}}=1 / 10$ and we 


\begin{tabular}{rccccccc}
\hline c & 0.1 & 0.3 & 0.5 & 0.7 & 0.8 & 0.9 & 0.95 \\
\hline SI & $7(0.23)$ & $13(0.42)$ & $22(0.73)$ & $42(1.39)$ & $66(2.20)$ & $138(4.92)$ & $281(9.64)$ \\
SAS & $5(0.50)$ & $8(0.71)$ & $12(1.09)$ & $21(1.87)$ & $31(2.90)$ & $53(4.84)$ & $56(4.95)$ \\
Jacobi & $8(0.67)$ & $14(1.15)$ & $22(1.92)$ & $40(3.34)$ & $60(4.87)$ & $104(9.07)$ & $110(9.20)$ \\
GS & $4(0.32)$ & $7(0.59)$ & $11(0.96)$ & $20(1.70)$ & $30(2.48)$ & $52(4.42)$ & $55(4.67)$ \\
SOR & $5(0.39)$ & $5(0.42)$ & $6(0.54)$ & $11(0.92)$ & $14(1.17)$ & $18(1.48)$ & $24(2.15)$ \\
$(\omega=0.93)$ & & & & & &
\end{tabular}

Table 1: Iterations number and CPU time in s (in bracket) at fixed $\sigma=100$

divided the unit disc in 100 regions. We study the behavior of Source Iteration (SI), Jacobi, SAS Gauss-Seidel (GS) and SOR with respect to $\sigma$ and $c$. At fixed $\sigma=100$, we set $\alpha=\sigma(1-c)$ and compare the $c$-dependence of the iterative methods. As shown by Table 1, all the method converge. It is observed that SOR method is efficient compared to the other methods. At fixed $c=0.95$, we set $\alpha=10$ and compare the $\sigma$-dependence of the method. The numerical results in Table 2 show the efficiency of the SOR method. It can be observed that SAS is comparable to GS, which is two time faster than Jacobi method. This confirms the theoretical results. We set $\alpha_{1}=\sigma$, $\alpha_{2}=\sigma \sqrt{(1-c)}, \omega_{a}=\frac{2}{1+\sqrt{1-\beta\left(\alpha_{1}\right)}}$ for $\sigma>15$ and $\omega_{a}=0.93$ for $\sigma \geq 15$. Comparative numerical results of SAS $\left(\alpha=\alpha_{1}\right)$, SAS $\left(\alpha=\alpha_{2}\right)$, SOR $\left(\alpha=\alpha_{1}, \omega=\omega_{a}\right)$ and SOR $\left(\alpha=\alpha_{2}, \omega=0.93\right)$ methods are plotted in Figure 1 at fixed $c=0.99(\sigma \in[1,100])$ and in Figure 2 at fixed $\sigma=100$ $(c \in[0.10 .99])$. It is observed that the SOR $\left(\alpha=\alpha_{2}, \omega=0.93\right)$ method is efficient compared to the other methods.

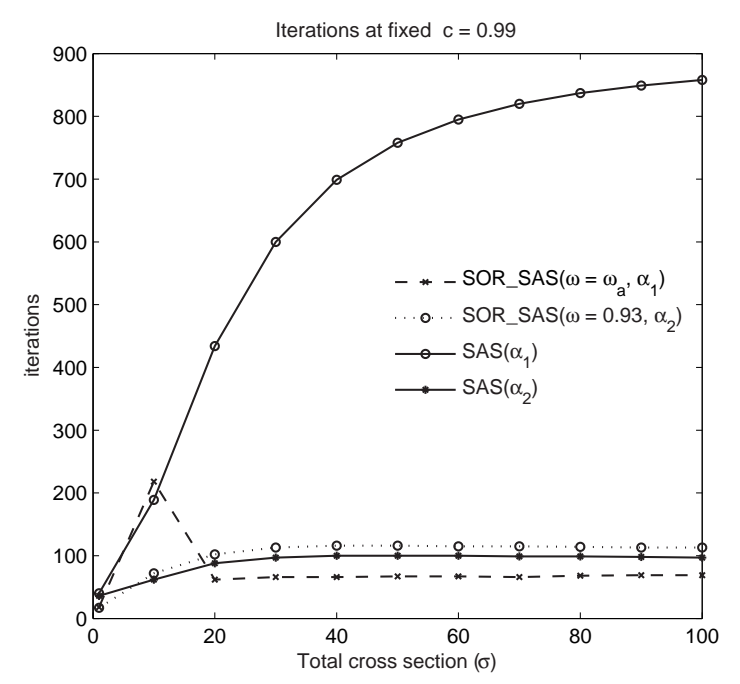

Figure 1: Comparison of the methods at fixed $c=0.99$, for $\sigma \in[1,100],(\epsilon=1 E-08)$.

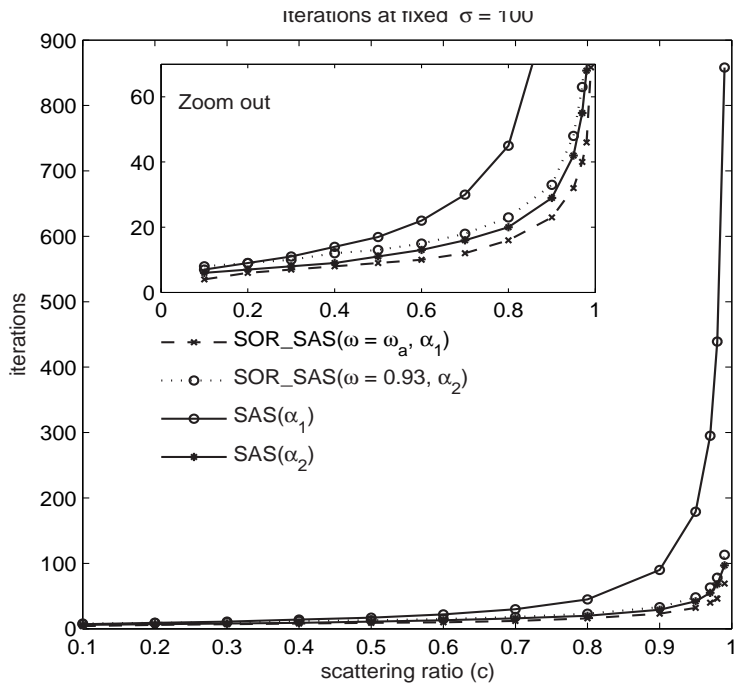

Figure 2: Comparison of the methods at fixed $\sigma=100$, for $c \in[0.1,0.99],(\epsilon=1 E-08)$. 


\begin{tabular}{rccccccc}
\hline$\sigma$ & 20 & 40 & 60 & 70 & 80 & 90 & 100 \\
\hline SI & $235(7.35)$ & $268(8.43)$ & $276(8.84)$ & $278(8.71)$ & $279(8.79)$ & $280(8.81)$ & $281(8.78)$ \\
SAS & $62(5.04)$ & $36(2.96)$ & $35(2.87)$ & $40(3.26)$ & $44(3.70)$ & $49(3.93)$ & $53(4.34)$ \\
Jacobi & $121(9.39)$ & $67(5.20)$ & $68(5.25)$ & $78(6.00)$ & $86(6.65)$ & $96(7.53)$ & $104(8.00)$ \\
GS & $61(4.82)$ & $35(2.73)$ & $34(2.60)$ & $39(3.09)$ & $43(3.34)$ & $48(3.67)$ & $52(4.09)$ \\
SOR & $71(5.46)$ & $41(3.15)$ & $28(2.14)$ & $24(1.89)$ & $21(1.60)$ & $19(1.48)$ & $19(1.50)$ \\
$(\omega=0.93)$ & & & & & & \\
\hline
\end{tabular}

Table 2: Iterations number and CPU time in s (in bracket) at fixed $c=0.95(\alpha=10)$

\section{Conclusion}

The analysis of a SOR acceleration of the SAS method yields convergence results similar to those obtained in finite dimensional linear systems with coefficient matrices possessing Property A. Previous numerical results have demonstrated the effectiveness of the SOR acceleration of the SAS method for solving the 2-D neutron transport problem.

\section{References}

[1] Awono Onana and J. Tagoudjeu. Iterative methods for a class of linear operator equations. Int. J. Contemp. Math. Sci., 4 (2009), No. 12, 549-564.

[2] O. Awono and J. Tagoudjeu. A splitting iterative method for solving the neutron transport equation. Math. Model. Anal., 14 (2009), No. 3, 271-289.

[3] O. Awono and J. Tagoudjeu. A self-adjoint and m-accretive splitting iterative method for solving the neutron transport equation in 1-D sphrical geometry. Proceeding of CARI'08 Rabat-Morocco, (2008), 331-338.

[4] P. Lascaux and R. Théodor. Analyse numérique matricielle appliquée à l'Art de l'Ingénieur. Volume 2, Masson, Paris, 1987.

[5] D. M. Young. Iterative solution of large linear systems. Academic Press, New York and London, 1971. 